\begin{tabular}{|c|c|c|}
\hline \multirow[t]{2}{*}{7} & $\begin{array}{l}\text { International Journal of Current Research in } \\
\text { Biosciences and Plant Biology }\end{array}$ & \\
\hline & Volume $4 \bullet$ Number 8 (August-2017) • ISSN: 2349-8080 (Online) & \\
\hline $\begin{array}{l}\text { EXCELLENT } \\
\text { PUBLISHERS } \\
\end{array}$ & Journal homepage: www.ijcrbp.com & wwwijecrip ocom \\
\hline
\end{tabular}

\title{
Statistical Study of Ionization for a Number of Oximes and Schiff Bases Theoretically by Using Molecular Modeling Programs
}

\author{
Mohammad Mahmoud Hussein Al-Niemi and Wisam Ibrahem AL-Shoraify*
}

Department of Chemistry, College of Education for Pure Sciences, Mosul University, Mosul, Iraq

*Corresponding author.

\begin{tabular}{|c|c|}
\hline Abstract & Article Info \\
\hline $\begin{array}{l}\text { In this study, the study included the calculation of fourteen ionic and aromatic } \\
\text { compounds, which included eight oximes and six Schiff bases. The parameters of the } \\
\text { ionization of the compounds under study were calculated at absolute temperature ( } 293 \mathrm{~K})\end{array}$ & $\begin{array}{l}\text { Accepted: } 30 \text { July } 2017 \\
\text { Available Online: 06 August } 2017\end{array}$ \\
\hline $\begin{array}{l}\text { using molecular modeling programs. Methods that included semi-empirical calculations } \\
\text { were AUSTEN MOMEL } 1 \text { (AM1), Hatree Fock (HF), and Density functional theory }\end{array}$ & Keywords \\
\hline $\begin{array}{l}\text { (DFT). The polarizable conductor calculation model (CPCM) was used to calculate the } \\
\text { physical variables in the solution phase instead of the gas phase using ethanol as a solvent. }\end{array}$ & $\begin{array}{l}\text { AUSTEN MOMEL } 1 \text { (AM1) } \\
\text { Density functional theory (DF }\end{array}$ \\
\hline The ionization constants were calculated using multiple Linear regression (MLR) analysis & Hatree Fock (HF) \\
\hline and using various descriptive variables selected to test their representatively of the & Oximes \\
\hline observed variation in $\mathrm{pK}_{\mathrm{a}}$ values These variables include a Mullikan charge on the atoms & Schiff base \\
\hline $\begin{array}{l}\text { of the hydroxyl and azomethine group }(\mathrm{C}=\mathrm{N}) \text {, the length of the double bond, the angles } \\
\text { values the value of the fixed dipole moment. DIHADRAL the total energy and the heat }\end{array}$ & \\
\hline of the formation temperature), molecular properties such as HOMO, LUMO, Molecular & \\
\hline hardness, electrolyte guide and chemical potential. These values were correlated with $\mathrm{pKa}$ & \\
\hline values. In this relationship, the values of $\mathrm{pK}_{\mathrm{a}}$ were calculated theoretically. The results & \\
\hline obtained showed that the HF method and the AM1 method were preferable to the EDP & \\
\hline method, although all methods were able to complete this type of calculation And with a & \\
\hline good efficiency in describing the effect of substituents on $\mathrm{pK}_{\mathrm{a}}$ values, these results were & \\
\hline $\begin{array}{l}\text { clearly indicated by the high correlation coefficients }\left(\mathrm{R}^{2}\right) \text { obtained from the regression } \\
\text { analysis and the correlation between the practical values of } \mathrm{pKa} \text { and those calculated }\end{array}$ & \\
\hline 1 is and the & \\
\hline
\end{tabular}

\section{Introduction}

The method of computational chemistry is of unusual methods compared with the previous methods in its mathematical rather than practical method. In arithmetic it does not require a sample, and in principle it is not restricted in terms of material conditions, but the accuracy of the values obtained depends entirely on the model Used to perform calculations. Since its inception in the early 1970s, with the development of programs for the calculation of molecular orbits and the development of the first methods of molecular mechanics in computational chemistry, many methods have been developed to 
estimate molecular properties computationally. Many of these methods have been evaluated to determine the parameters of acid dissociation and the most accurate results available from computation, which is called $a b$ initio, means that these calculations are usually oriented towards a precise mathematical solution; the equations used to describe the system are derived entirely from theoretical principles (da Silva et al., 1999). The software of physics and theoretical chemistry (Perrin et al., 1981) is defined as a science that deals with the application of theoretical and mathematical principles in solving problems. Computational chemistry is one of the branches of physical chemistry (Barlin and Perrin, 1972). The molecular modeling software is one of the most important applications of this science, and finds a number of important characteristics.

The approximation methods used to solve the Schrödinger equation for physically and chemically important systems will usually lead to complex calculations that could not be achieved solved. Quantitative theoretical calculations were disabled because of the complexities involved in solving mathematical problems. The cost was high for large calculations as well as the slow calculation speed, which could last for months to solve a relatively simple molecule and years to solve medium molecules.

\section{Materials and methods}

\section{Theoretical calculations}

The lowest molecular energy level of the compounds under study was calculated using semi-experimental AM1 methods, DFT B3LYP, bases set 3.21G and basic set calculations $6.311 \mathrm{G}(\mathrm{d}, \mathrm{p}) \mathrm{HF}$. The following values were calculated: lowest molecular energy, bipolar and charge and the length of the dihedral angle, the angle values $(1,2,7$ and $8,7,9)$, the higher molecular occupancy values (HOMO), and the low-molecularactivated (LUMO) orbitral. HOMO and LUMO values were used to calculate some of the properties of the molecule such as Hardness (Parr and Pearson, 1983), Electronic Chemical Potential (Brouwer, 2003) and Global Electrophilicity Index (Szabo and Nostlund, 1989) and were found through the following relationships.

$$
\begin{aligned}
& \text { H }=(\text { ELUMO }- \text { E HOMO }) \ldots(1) \\
& \text { M }=(\text { EHOMO }+ \text { ELUMO }) \ldots(2) \\
& W=\left(\mu^{\wedge} 2 / \eta\right) \ldots(3)
\end{aligned}
$$

These values were computed by GAUSSIAN 09 and Gaussian Program Gauss View (5.0.8). The calculation of the constant ionization of the compounds under study (Table 1).

Table 1. The names and structures and values of the ionization constant of the compounds.

Number




Compound

\section{Multiple linear regression method}

MLR is an advanced statistical technique that ensures the accuracy of inference to improve search results by optimizing the use of data in the creation of causal relationships between phenomena (subject of research).

Multiple linear regression is a mathematical equation that expresses the relationship between two variables and is used to estimate past values and to predict future values. It is also a regression of the dependent variable (Y) on many independent variables $\mathrm{X} 1, \mathrm{X} 2, \ldots \mathrm{XK}, \mathrm{We}$ can predict the value of the digital $\mathrm{pKa}$ ionization constant based on the values of the Mullikan charge, the length of the signal and other values. Multiple linear regression is not just one method but a set of methods that can be used to determine the relationship between a continuous dependent variable and a number of independent variables that are usually continuous.

The linear equation in multiple linear regression is:

$$
\mathrm{Y}=\mathrm{a}+\mathrm{b} 1 \mathrm{X} 1+\mathrm{b} 2 \mathrm{X} 2+\ldots \ldots \ldots+\mathrm{e} \ldots \ldots . .(4)
$$

The proper and correct assessment of the correlation between variables can be inferred by Standard Deviation (SD) (Isaacs, 1969) and Correlation Coefficient (R) values. A large number of ready-made calculator programs are now available and can be used for this function in more difficult tasks such as Multiple Regression Analysis, the independent variable is not sufficient to illustrate experimental results. It is often necessary to use more than one independent variable in the correlation analysis to describe or describe a particular system. In this case, we will speak of a type of regression analysis called regression analysis die.
Number of variables (Multiple Parametric Regression Analysis) The Multivariate Linear Regression Equation of a Y Variable is a series of independent variables. After obtaining the results of the regression equation, we must show whether these transactions are statistically acceptable, which is statistically significant, noting that the morality is for each factor separately.

In order to judge the significance of regression coefficients, we use the $\mathrm{T}$ test and the corresponding probability level. SPSS will automatically extract the T test and the corresponding probability level.

Statistics have also been used to determine the overall morale of the model, including $\left(\mathrm{R}^{2}\right),\left(\mathrm{R}^{-2}\right),(\mathrm{R})$.

\section{Results and discussion}

The compounds under study were developed in the form of a single group and the calculation of some physical variables for them and the effective sites in them and then finding the relationship between them and the values of ionization constants pKa. Ionization of ionic compounds can be affected by the charge found on the $\mathrm{O}(\mathrm{O})$ and Halogen $(\mathrm{X})$ atoms. Thus, the values of $\mathrm{pKa}$ are determined by the effect of the compensated groups in the reaction centers represented by the $\mathrm{R}$ group that will affect these values through two effects: the first is the induction effect of these groups; and the second: the values of the spatial disability that these groups limit their size, and that these two effects can change the value of electronic charge on the atoms of the center of the interaction. The values of these compounds were measured at $(293 \mathrm{~K})$ and in the above three methods as follows: 
Table 2. The values obtained theoretically in the (AM1) method.

\begin{tabular}{|c|c|c|c|c|c|c|c|c|c|c|c|c|}
\hline $\begin{array}{c}\text { Compd. } \\
\text { AM1 }\end{array}$ & Charge C7 & $\begin{array}{c}\text { Charge } \\
\text { CH3 or H }\end{array}$ & Charge N9 & Charge 010 & Charge H11 & DM & Angle 127 & Angle 2 & Dihedral & Bond CN & H formation & ET(et) \\
\hline 1 & 0.015 & -0.258 & -0.072 & -0.248 & 0.249 & 3.162 & 111.924 & 109.295 & 4.270 & 1.239 & 8.263 & 4.091 \\
\hline 2 & -0.086 & -0.181 & -0.009 & -0.256 & 0.255 & 5.835 & 121.083 & 119.414 & -155.452 & 1.341 & 6.239 & 2.081 \\
\hline 3 & -0.082 & 0.132 & -0.057 & -0.302 & 0.271 & 0.991 & 119.999 & 120.000 & 0.008 & 1.260 & 3.951 & 0.089 \\
\hline 4 & -0.074 & 0.189 & -0.069 & -0.275 & 0.249 & 0.903 & 119.999 & 119.996 & -179.998 & 1.260 & 5.174 & 1.330 \\
\hline 5 & -0.069 & 0.128 & -0.075 & -0.305 & 0.268 & 1.282 & 120.004 & 120.000 & -0.012 & 1.260 & 3.914 & 0.051 \\
\hline 6 & -0.062 & 0.186 & -0.088 & -0.278 & 0.246 & 1.479 & 119.999 & 119.996 & -179.998 & 1.260 & 5.134 & 1.288 \\
\hline 7 & -0.069 & 0.128 & -0.074 & -0.305 & 0.269 & 1.543 & 120.004 & 120.000 & 0.003 & 1.260 & 5.335 & 0.635 \\
\hline 8 & -0.063 & 0.186 & -0.086 & -0.278 & 0.246 & 1.751 & 120.004 & 119.997 & 180.000 & 1.201 & 6.558 & 1.876 \\
\hline 9 & 0.046 & -0.271 & -0.135 & -0.259 & 0.247 & 4.418 & 118.099 & 119.998 & -0.002 & 1.260 & 11.364 & 4.794 \\
\hline 10 & 0.069 & -0.275 & -0.153 & -0.268 & 0.248 & 5.471 & 118.099 & 119.998 & -0.007 & 1.260 & 11.292 & 4.722 \\
\hline 11 & 0.049 & -0.271 & -0.142 & -0.268 & 0.247 & 4.193 & 118.099 & 119.997 & 0.007 & 1.260 & 11.295 & 4.724 \\
\hline 12 & 0.073 & -0.276 & -0.155 & 0.044 & 0.000 & 5.886 & 118.099 & 119.997 & -0.003 & 1.260 & 13.035 & 6.860 \\
\hline 13 & 0.069 & -0.275 & -0.152 & -0.028 & 0.000 & 5.754 & 118.099 & 120.001 & -0.003 & 1.260 & 13.052 & 6.860 \\
\hline 14 & 0.050 & -0.271 & -0.142 & -0.203 & 0.000 & 4.082 & 118.099 & 119.997 & 0.007 & 1.260 & 12.564 & 6.361 \\
\hline $\begin{array}{c}\text { ЕHOMO } \\
\text { (et) }\end{array}$ & $\begin{array}{c}\text { ELUMO } \\
\text { (et) }\end{array}$ & $\eta$ & $\mu$ & $\mathbf{W}$ & & & & & & & & \\
\hline-9.695 & -0.700 & -4.497 & 5.197 & -3.003 & & & & & & & & \\
\hline-9.426 & -0.670 & -4.378 & 5.048 & -2.910 & & & & & & & & \\
\hline-9.199 & -0.494 & -4.353 & 4.847 & -2.698 & & & & & & & & \\
\hline-9.153 & -0.427 & -4.363 & 4.790 & -2.629 & & & & & & & & \\
\hline-8.971 & -0.376 & -4.297 & 4.673 & -2.541 & & & & & & & & \\
\hline-9.001 & -0.315 & -4.343 & 4.658 & -2.498 & & & & & & & & \\
\hline-8.975 & -0.390 & -4.292 & 4.682 & -2.554 & & & & & & & & \\
\hline-9.007 & -0.328 & -4.339 & 4.668 & -2.511 & & & & & & & & \\
\hline-8.822 & -1.013 & -3.904 & 4.918 & -3.097 & & & & & & & & \\
\hline-8.947 & -1.072 & -3.937 & 5.009 & -3.187 & & & & & & & & \\
\hline-8.701 & -1.004 & -3.848 & 4.852 & -3.059 & & & & & & & & \\
\hline-9.076 & -1.159 & -3.958 & 5.118 & -3.309 & & & & & & & & \\
\hline-9.034 & -1.131 & -3.951 & 5.083 & -3.269 & & & & & & & & \\
\hline-8.707 & -1.008 & -3.849 & 4.858 & -3.065 & & & & & & & & \\
\hline
\end{tabular}


Table 3. The values obtained theoretically in the (DFT) method.

\begin{tabular}{|c|c|c|c|c|c|c|c|c|c|c|c|c|}
\hline $\begin{array}{l}\text { Compd. } \\
\text { DFT }\end{array}$ & $\begin{array}{l}\text { Charge } \\
\text { C7 }\end{array}$ & $\begin{array}{l}\text { Charge } \\
\text { CH3 or H }\end{array}$ & Charge N9 & $\begin{array}{l}\text { Charge } \\
010\end{array}$ & $\begin{array}{l}\text { Charge } \\
\text { H11 }\end{array}$ & DM & $\begin{array}{l}\text { Angle } \\
127\end{array}$ & Angle 2 & Dihedral & $\begin{array}{l}\text { Bond } \\
\mathrm{CN}\end{array}$ & H formation & ET (et) \\
\hline 1 & 0.3 & -0.58 & -0.259 & -0.484 & 0.426 & 2.0074 & 118.099 & 119.998 & -0.006 & 1.25999 & -12340.5 & -12344.6 \\
\hline 2 & 0.31 & -0.599 & -0.322 & -0.43 & 0.38 & 3.2904 & 119.695 & 112.435 & 179.336 & 1.2969 & -12341.8 & -12346 \\
\hline 3 & 0.09 & 0.213 & -0.231 & -0.472 & 0.413 & 2.4595 & 120 & 120 & 0 & 1.2936 & -12879.5 & -12883.2 \\
\hline 4 & 0.093 & 0.241 & -0.289 & -0.442 & 0.395 & 1.1641 & 123.705 & 111.504 & 179.999 & 1.29167 & -12880 & -12883.8 \\
\hline 5 & 0.113 & 0.222 & -0.3 & -0.45 & 0.385 & 2.319 & 121.48 & 120.791 & 0.009 & 1.28942 & -12880 & -12883.8 \\
\hline 6 & 0.088 & 0.236 & -0.298 & -0.447 & 0.391 & 2.096 & 124.495 & 111.493 & -179.993 & 1.29272 & -12880.1 & -12883.9 \\
\hline 7 & 0.113 & 0.222 & -0.3 & -0.449 & 0.385 & 2.7096 & 121.589 & 120.741 & -0.004 & 1.2895 & -13943 & -13947.6 \\
\hline 8 & 0.087 & 0.235 & -0.298 & -0.447 & 0.391 & 3.023 & 117.45 & 111.467 & -179.999 & 1.29257 & -13943.1 & -13947.7 \\
\hline 9 & 0.352 & -0.629 & -0.705 & -0.641 & 0.371 & 6.3161 & 116.341 & 125.623 & 0.642 & 1.28361 & -18593.8 & -18600.3 \\
\hline 10 & 0.319 & -0.625 & -0.613 & -0.619 & 0.381 & 5.3549 & 117.269 & 125.462 & -5.022 & 1.28307 & -18593.5 & -18600 \\
\hline 11 & 0.315 & -0.625 & -0.622 & -0.619 & 0.381 & 5.6904 & 117.322 & 125.765 & -0.5641 & 1.28465 & -18593.5 & -18600 \\
\hline 12 & 0.232 & -0.981 & -0.599 & 0.182 & 0 & 8.2861 & 119.766 & 120 & 0 & 1.2936 & -86245.1 & -86250.9 \\
\hline 13 & 0.322 & -0.625 & -0.612 & 0.055 & 0 & 7.3123 & 117.084 & 125.264 & 4.832 & 1.28301 & -29004.7 & -29010.9 \\
\hline 14 & 0.341 & -0.694 & -0.603 & -0.547 & 0 & 5.1574 & 119.766 & 120 & 0 & 1.2936 & -19652.1 & -19659.4 \\
\hline $\begin{array}{l}\text { EHOMO } \\
\text { (et) }\end{array}$ & $\begin{array}{l}\text { ELUMO } \\
\text { (et) }\end{array}$ & $\eta$ & $\mu$ & $\mathbf{W}$ & & & & & & & & \\
\hline-6.13671 & -1.27812 & -2.4293 & 3.707417 & -2.82899 & & & & & & & & \\
\hline-6.42135 & -1.45799 & -2.48168 & 3.939666 & -3.12711 & & & & & & & & \\
\hline-6.22461 & -1.13281 & -2.5459 & 3.678709 & -2.65778 & & & & & & & & \\
\hline-6.15386 & -1.32601 & -2.41392 & 3.739935 & -2.89717 & & & & & & & & \\
\hline-5.86106 & -1.08356 & -2.38875 & 3.472311 & -2.52369 & & & & & & & & \\
\hline-5.97916 & -1.07213 & -2.45352 & 3.525645 & -2.53313 & & & & & & & & \\
\hline-5.83249 & -1.10342 & -2.36453 & 3.467957 & -2.54315 & & & & & & & & \\
\hline-5.94134 & -1.08138 & -2.42998 & 3.511359 & -2.53699 & & & & & & & & \\
\hline-5.7631 & -1.9331 & -1.915 & 3.8481 & -3.86628 & & & & & & & & \\
\hline-5.85943 & -1.69554 & -2.08194 & 3.777487 & -3.42694 & & & & & & & & \\
\hline-5.54133 & -1.64112 & -1.9501 & 3.591225 & -3.30672 & & & & & & & & \\
\hline-5.11738 & -1.73418 & -1.69275 & 3.428121 & -3.47127 & & & & & & & & \\
\hline-6.0744 & -1.78371 & -2.14681 & 3.93174 & -3.60035 & & & & & & & & \\
\hline-6.40747 & -1.73119 & -2.33974 & 4.07211 & -3.54358 & & & & & & & & \\
\hline
\end{tabular}


Table 4. The values obtained theoretically in the HF method.

\begin{tabular}{|c|c|c|c|c|c|c|c|c|c|c|c|c|}
\hline $\begin{array}{l}\text { Compd } \\
\text { HF }\end{array}$ & $\begin{array}{l}\text { Charge } \\
\text { C7 }\end{array}$ & $\begin{array}{l}\text { Charge } \\
\text { CH3 or } \\
\text { H }\end{array}$ & $\begin{array}{l}\text { Charg } \\
\text { e N9 }\end{array}$ & $\begin{array}{l}\text { Charge } \\
\text { O10 }\end{array}$ & $\begin{array}{l}\text { Charge } \\
\text { H11 }\end{array}$ & DM & $\begin{array}{l}\text { Angle } \\
127\end{array}$ & Angle 2 & Dihedral & $\begin{array}{l}\text { Bond } \\
\mathrm{CN}\end{array}$ & $\mathbf{H}$ forma-tion & ET(et) \\
\hline 1 & 0.103 & -0.168 & -0.181 & -0.406 & 0.293 & 3.7103 & 116.819 & 121.678 & 0.001 & 1.25757 & -12337.2 & -12341.5 \\
\hline 2 & 0.08 & -0.247 & -0.149 & -0.413 & 0.335 & 7.0197 & 121.083 & 119.414 & -155.452 & 1.34078 & -12335.6 & -12339.8 \\
\hline 3 & 0.249 & 0.113 & -0.187 & -0.395 & 0.295 & 0.6221 & 121.537 & 120.347 & 0 & 1.25094 & -12877.5 & -12881.5 \\
\hline 4 & 0.242 & 0.137 & -0.153 & -0.461 & 0.338 & 1.9503 & 119.999 & 119.996 & -179.998 & 1.26003 & -12875.2 & -12879.2 \\
\hline 5 & 0.255 & 0.108 & -0.203 & -0.401 & 0.292 & 1.8999 & 122.524 & 119.965 & -0.005 & 1.252 & -12877.5 & -12881.6 \\
\hline 6 & 0.216 & 0.131 & -0.211 & -0.411 & 0.131 & 1.9519 & 126.119 & 112.245 & -180 & 1.25688 & -12877.4 & -12881.5 \\
\hline 7 & 0.253 & 0.108 & -0.282 & -0.401 & 0.292 & 2.4112 & 122.709 & 11.943 & 0 & 1.25201 & -13938.6 & -13943.5 \\
\hline 8 & 0.214 & 0.13 & -0.211 & -0.411 & 0.299 & 2.5026 & 126.203 & 112.225 & 179.999 & 1.25697 & -13938.5 & -13943.4 \\
\hline 9 & 0.189 & -0.241 & -0.422 & -0.494 & 0.289 & 4.8281 & 117.054 & 125.966 & -1.574 & 1.25419 & -18583.5 & -18590.3 \\
\hline 10 & 0.173 & -0.247 & -0.416 & -0.482 & 0.29 & 6.0395 & 117.16 & 125.983 & 0.496 & 1.25405 & -18583.6 & -18590.4 \\
\hline 11 & 0.169 & -0.257 & -0.422 & -0.489 & 0.287 & 4.3311 & 117.309 & 126.453 & 2.181 & 1.2549 & -18583.5 & -18590.4 \\
\hline 12 & 0.175 & -0.25 & -0.417 & -0.068 & 0 & 7.1535 & 117.159 & 125.974 & 1.161 & 1.25479 & -86528.1 & -86534.5 \\
\hline 13 & 0.173 & -0.18 & -0.465 & -0.131 & 0 & 4.8226 & 116.499 & 126.368 & -1.483 & 1.25666 & -29034.4 & -29040.8 \\
\hline 14 & 0.107 & -0.227 & -0.367 & -0.468 & 0 & 5.2822 & 118.099 & 119.997 & 0.007 & 1.26 & -19641.4 & -19649.1 \\
\hline $\begin{array}{l}\text { EHOM } \\
\text { O(et) }\end{array}$ & $\begin{array}{l}\text { ELUMO( } \\
\text { et) }\end{array}$ & $\eta$ & $\mu$ & \multicolumn{2}{|l|}{$\mathbf{W}$} & & & & & & & \\
\hline-9.13732 & 2.321949 & -5.72963 & 3.407684 & \multicolumn{2}{|c|}{-1.01336} & & & & & & & \\
\hline-8.69187 & 1.91541 & -5.30364 & 3.388227 & \multicolumn{2}{|c|}{-1.08228} & & & & & & & \\
\hline-8.71962 & 2.34399 & -5.53181 & 3.187816 & \multicolumn{2}{|c|}{-0.91852} & & & & & & & \\
\hline-8.41621 & 2.341813 & -5.37901 & 3.0372 & \multicolumn{2}{|c|}{-0.85746} & & & & & & & \\
\hline-8.43036 & 2.71216 & -5.57126 & 2.859102 & \multicolumn{2}{|c|}{-0.73363} & & & & & & & \\
\hline-8.54057 & 2.709439 & -5.625 & 2.915565 & \multicolumn{2}{|c|}{-0.7556} & & & & & & & \\
\hline-8.36669 & 2.673248 & -5.51997 & 2.846721 & \multicolumn{2}{|c|}{-0.73405} & & & & & & & \\
\hline-8.47662 & 2.677874 & -5.57725 & 2.899375 & \multicolumn{2}{|c|}{-0.75363} & & & & & & & \\
\hline-8.3637 & 2.301812 & -5.33275 & 3.030942 & \multicolumn{2}{|c|}{-0.86134} & & & & & & & \\
\hline-8.50383 & 2.406304 & -5.45507 & 3.048765 & \multicolumn{2}{|c|}{-0.85196} & & & & & & & \\
\hline-8.15254 & 2.390794 & -5.27166 & 2.880871 & \multicolumn{2}{|c|}{-0.78717} & & & & & & & \\
\hline-8.66384 & 2.31569 & -5.49352 & 3.176243 & \multicolumn{2}{|c|}{-0.91822} & & & & & & & \\
\hline-8.71064 & 2.17664 & -5.44736 & 3.269234 & \multicolumn{2}{|c|}{-0.98102} & & & & & & & \\
\hline-7.58545 & 1.415809 & -4.50371 & 3.086929 & \multicolumn{2}{|c|}{-1.05792} & & & & & & & \\
\hline
\end{tabular}


An example of this is compound number one, which is illustrated as follows:

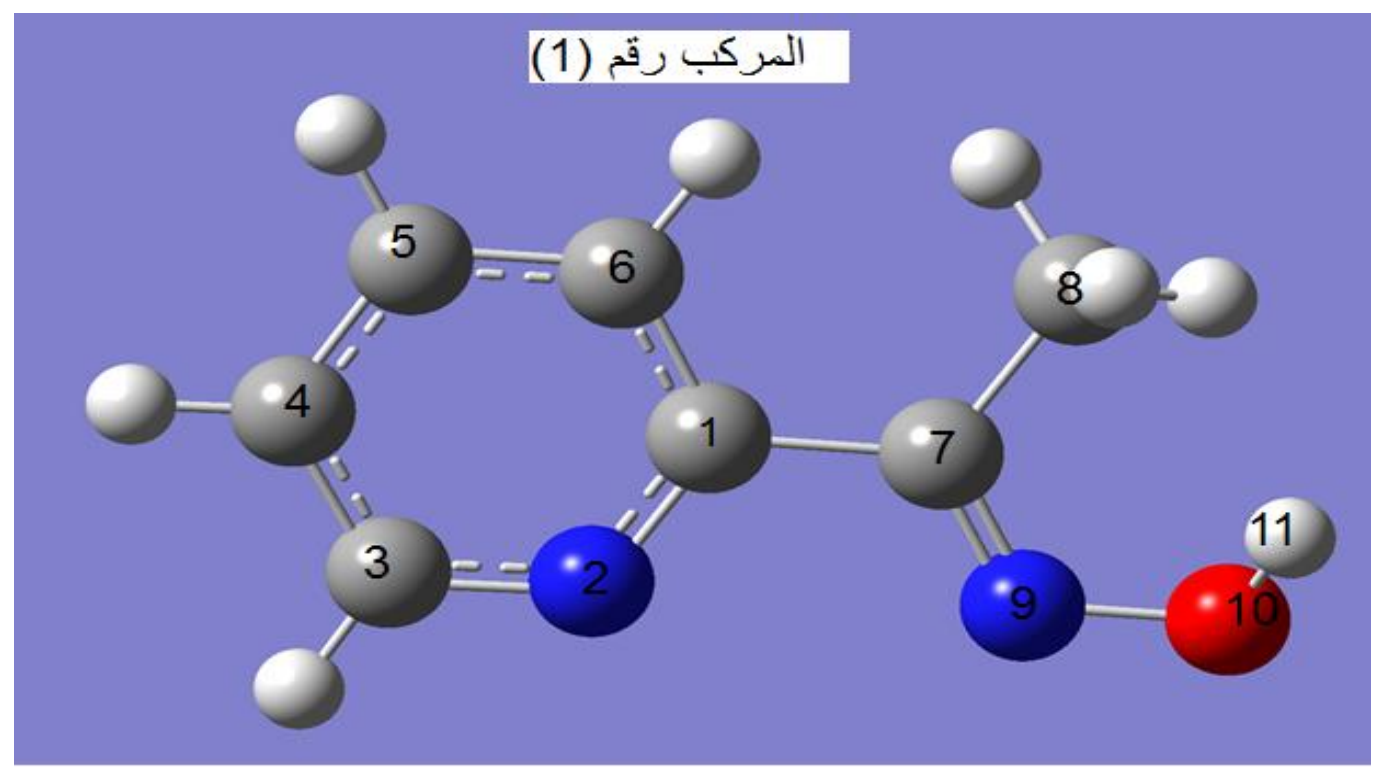

These variables have been included and chosen for accuracy and validity and therefore rely on them in statistical analysis, so that the correct statistical analysis must have the basic conditions is to obtain a correlation coefficient strong and close to one and be a match between practical and theoretical values and that the values are consistent with the chemical principles and principles For each effect, the relationship between each variable was then determined with the constants and then the variables were added sequentially in the input and deletion method to determine the sensitivity of these variables and their applicability to calculate the pKa ionization constant is given below. In considering the above tables, it may be noted that:

Table 5. Correlation coefficient values between variables calculated by AM1 method at (K293)

\begin{tabular}{|c|c|c|c|c|c|c|c|c|c|c|c|c|c|c|c|c|c|}
\hline & charge7C & 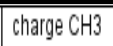 & chargeng & charge010 & \begin{tabular}{|c|} 
chargeH11 \\
\end{tabular} & angle127 & angle 2 & dihadral & duble CN & 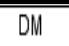 & EHOMO & $\begin{array}{l}\text { ELUMO } \\
\end{array}$ & \begin{tabular}{|l|} 
hardness \\
\end{tabular} & potential & electrophilicity & Hfformation & 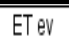 \\
\hline charge7c & 1 & $.864^{\prime \prime}$ & $.886^{\prime \prime \prime}$ & $.624^{x}$ & $.631^{n}$ & $.556^{1}$ & .088 & .289 & -.144 & $.741^{11}$ & .337 & $.933^{110}$ & $.836^{111}$ & $.603^{x}$ & $.901^{11}$ & $.963^{111}$ & $.943^{\mathrm{m}}$ \\
\hline charge $\mathrm{CH}_{3}$ & $.864^{\prime \prime}$ & 1 & $.656^{\circ}$ & .526 & .510 & $.563^{\circ}$ & .243 & .128 & .251 & $.898^{\prime \prime}$ & .059 & $.934^{\prime \prime \prime}$ & $.683^{\prime \prime}$ & $.805^{\prime \prime \prime}$ & $.951^{\prime \prime}$ & $.877^{\prime \prime}$ & $.879^{\prime \prime}$ \\
\hline chargeng & $.886^{\prime \prime}$ & $.656^{\prime}$ & 1 & $.540^{\circ}$ & $.588^{\circ}$ & .331 & -156 & .059 & .070 & .532 & $.537^{\prime \prime}$ & $.813^{\prime \prime \prime}$ & $.864^{\prime \prime}$ & .348 & $.737^{\prime \prime}$ & $.813^{\prime \prime \prime}$ & $\cdot .769^{\prime \prime}$ \\
\hline charge010 & $.624^{\circ}$ & .526 & $.540^{\circ}$ & 1 & $.873^{\prime \prime \prime}$ & .215 & .051 & .093 & .029 & $.642^{\circ}$ & .006 & $.659^{\prime}$ & .462 & $.594^{\prime}$ & $.693^{\prime \prime}$ & $.688^{\prime \prime}$ & $.752^{\prime \prime}$ \\
\hline chargetH11 & $.631^{\prime}$ & .510 & $.588^{\circ}$ & $.873^{\prime \prime}$ & 1 & .169 & -139 & -.131 & .017 & $.549^{x}$ & .235 & $.644^{\circ}$ & $.579^{\prime \prime}$ & .415 & $.628^{n}$ & $.723^{\prime \prime \prime}$ & $\cdot .780^{\mathrm{m}}$ \\
\hline angle127 & $.556^{\circ}$ & $.563^{\circ}$ & .331 & .215 & .169 & 1 & $.863^{\prime \prime}$ & .236 & .318 & .254 & .375 & .401 & .070 & $.636^{\prime \prime}$ & .487 & .429 & -499 \\
\hline angle 2 & -.088 & .243 & -156 & .051 & .139 & $.863^{\prime \prime}$ & 1 & .064 & .173 & .006 & $.725^{\mathrm{m}}$ & .020 & .417 & -.508 & .120 & .018 & .088 \\
\hline dihadral & .289 & -128 & .059 & .093 & .131 & .236 & .064 & 1 & $.675^{\prime \prime}$ & .041 & .227 & .157 & .235 & .022 & -120 & .269 & .212 \\
\hline duble CN & -144 & -251 & .070 & .029 & .017 & .318 & .173 & $.675^{\prime \prime}$ & 1 & .411 & -196 & .160 & .003 & .288 & .197 & .048 & -034 \\
\hline DM & $.741^{\prime \prime}$ & $.898^{\prime \prime}$ &. .532 & $.642^{\prime \prime}$ & $-.549^{n}$ & -254 & .006 & .041 & .411 & 1 & .046 & $.878^{\prime \prime \prime}$ & $.637^{\circ}$ & $.764^{\prime \prime}$ & $.900^{\prime \prime}$ & $.819^{\prime \prime}$ & $.820^{\mathrm{m}}$ \\
\hline EHOMO & .337 & .059 & $.537^{7}$ & .006 & .235 & .375 & $.725^{\prime \prime}$ & .227 & .196 & .046 & 1 & .267 & $.742^{\prime \prime}$ & .484 & .076 & .363 & .234 \\
\hline ELUMO & $.933^{\prime \prime \prime}$ & $.934 "$ & $.813^{\prime \prime}$ & $.659^{\prime \prime}$ & $.644^{n}$ & .401 & .020 & .157 & -160 & $.878^{\prime \prime}$ & -267 & 1 & $.844^{\prime \prime}$ & $.714^{\prime \prime}$ & $.981^{\prime \prime}$ & $.950^{\prime \prime}$ & $.930^{\prime \prime}$ \\
\hline hardness & $.836^{\prime \prime}$ & $.683^{\prime \prime}$ & $.864^{\prime \prime}$ & .462 & $-.579^{x}$ & .070 & .417 & .235 & .003 & $.637^{\circ}$ & $.742^{\prime \prime}$ & $.844^{\prime \prime}$ & 1 & .227 & $-.725^{\prime \prime}$ & $.863^{\prime \prime}$ & $.777^{\prime \prime}$ \\
\hline potential & $.603^{\prime}$ & $.805^{\prime \prime}$ & .348 & $.594^{\prime \prime}$ & .415 & $.636^{\circ}$ & .508 & -022 & .288 & $.764^{\prime \prime}$ & .484 & $.714^{\prime \prime}$ & .227 & 1 & $.835^{\prime \prime}$ & $.599^{\circ}$ & $.674^{\prime \prime}$ \\
\hline electrophilicity & $.901^{\prime \prime}$ & $.951^{\prime \prime}$ & $.737^{\prime \prime}$ & $.693^{\prime \prime}$ & $.628^{\circ}$ & .487 & .120 & -120 & .197 & $.900 "$ & .076 & $.981^{\prime \prime}$ & $-725^{\prime \prime}$ & $.835^{\prime \prime}$ & 1 & $.913^{\prime \prime \prime}$ & $.918^{\prime \prime}$ \\
\hline Hformation & $.963^{\prime \prime}$ & $.877^{\prime \prime}$ & $.813^{\prime \prime \prime}$ & $.688^{\prime \prime}$ & $.723^{\prime \prime \prime}$ & .429 & .018 & .269 & .048 & $.819^{\prime \prime}$ & .363 & $.950^{\prime \prime}$ & $.863^{\mathrm{m}}$ & $.599^{\circ}$ & $.913^{\prime \prime}$ & 1 & $.984^{\prime \prime}$ \\
\hline ET ev & $.943^{\prime \prime}$ & $.879^{\prime \prime}$ & $.769^{\prime \prime}$ & $.752^{\prime \prime}$ & .7800 & -499 & .088 & .212 & .034 & $.820^{\prime \prime}$ & .234 & $.930^{\prime \prime}$ & $.777^{\prime \prime}$ & $.674^{\prime \prime}$ & $.918^{\prime \prime}$ & $.984^{\prime \prime \prime}$ & 1 \\
\hline
\end{tabular}


Table 6. Results of the statistical analysis of the relationship between the values of pKa and the variables calculated by( AM1) at $(293 \mathrm{~K})$

\begin{tabular}{lllllll}
\hline Model & R & R Square & Adjusted R Square & Std. Error of the estimate & F & Sig. \\
\hline 1 & $0.997^{\mathrm{a}}$ & 0.995 & 0.966 & 0.6601793 & 34.156 & $0.029^{\mathrm{a}}$ \\
\hline
\end{tabular}

\begin{tabular}{lll}
\hline Coefficients & B \\
\hline 1 & (Constant) & -76.240 \\
& charge6C & -53.005 \\
charge CH3 & 114.979 \\
chargeN9 & -51.929 \\
chargeO10 & -22.167 \\
chargeH11 & 75.306 \\
angle 2 & -2.846 \\
dihadral & .069 \\
duble CN & 392.027 \\
DM & 2.237 \\
EHOMO & 13.590 \\
ET ev & 7.777 \\
\hline
\end{tabular}

COMPUTE PKA=-76.240+-53.005*charge6C $+114.979 *$ chargeCH3+51.929*chargeN9 +-22.167*chargeO10 +75.306*chargeH11 +$2.846 *$ angle $2+.069 *$ dihadral $+392.027 *$ dubleCN $+2.237 * \mathrm{DM}+13.590 * \mathrm{EHOMO}+7.777 * \mathrm{ETev}$.

Table 7. Correlation coefficient values between variables calculated by (HF) method at (K293)

\begin{tabular}{|c|c|c|c|c|c|c|c|c|c|c|c|c|c|c|c|c|c|}
\hline 293 & Irge7c & $\mathrm{gec} \mathrm{CH} 3$ & chargeng & charge010 & chargetH11 & $\begin{array}{l}\text { angle1 } 27 \\
\end{array}$ & angle 2 & |dihadral & blecN & DM & EHOMO & ELLUMO & ardness & potential & electrophilicity & H Hormation & $\overline{E \text { ETeV }}$ \\
\hline charge7C & 1 & $.787^{\prime \prime}$ & .163 & .015 & .238 & .493 & .366 & .084 & $.578^{1}$ & $.748^{\mathrm{m}}$ & .016 & $.726^{\mathrm{m}}$ & .423 & $.682^{113}$ & $.804^{11}$ & 100 & .100 \\
\hline charge $\mathrm{CH}_{3}$ & $.787^{\prime \prime}$ & 1 & $.656^{\circ}$ & 145 & .314 & $.812^{\prime \prime}$ & .404 & .058 & .284 & $.898^{\prime \prime}$ & .086 & $.609^{\prime}$ & .414 & .482 & $.599^{\prime \prime}$ & .388 & .388 \\
\hline chargeng & .163 & $.656^{\circ}$ & 1 & .310 & $.548^{\prime}$ & $.644^{\circ}$ & 139 & .326 & .349 & $.553^{\prime}$ & .330 & .227 & .330 & .091 & .042 & .491 & .491 \\
\hline charge 010 & .015 & 145 & .310 & 1 & $.667^{\prime \prime}$ & .201 & .070 & .109 & .063 & .303 & .380 & .004 & -233 & .348 & .207 & $.788^{\prime \prime}$ & . $788^{\prime \prime}$ \\
\hline charget11 & .238 & .314 & $.548^{\circ}$ & $.667^{\prime \prime}$ & 1 & .269 & 173 & .046 & .204 & .371 & .242 & .416 & .388 & .164 & .323 & $.623^{\circ}$ & $.623^{\prime}$ \\
\hline angle1 27 & .493 & $.812^{\prime \prime}$ & $.644^{\prime}$ & .201 & .269 & 1 & .368 & .036 & .074 & $.610^{\circ}$ & . 003 & .518 & .306 & .481 & $.539^{\circ}$ & .371 & .371 \\
\hline angle 2 & .366 & .404 & 139 & .070 & 173 & .368 & 1 & .058 & .095 & .289 & .098 & .317 & .130 & .381 & .384 & 1711 & -171 \\
\hline dihadral & .084 & .058 & .326 & .109 & .046 & .036 & .058 & 1 & .431 & .013 & .078 & .140 & .038 & 199 & .194 & 131 & .131 \\
\hline duble CN & $.578^{\prime}$ & .284 & .349 & .063 & .204 & .074 & .095 & .431 & 1 & .455 & 144 & .403 & .155 & .500 & .529 & .132 & .132 \\
\hline DM & $.748^{\prime \prime}$ & $.898^{\prime \prime}$ & $.553^{\prime}$ & .303 & .371 & $.610^{\prime}$ & .289 & .013 & .455 & 1 & .064 & $.553^{\prime}$ & .366 & .451 & $.558^{\prime}$ & $.538^{\circ}$ & $.538^{\circ}$ \\
\hline EHOMO & .016 & .086 & .330 & .380 & .242 & .003 & .098 & .078 & 144 & .064 & 1 & .407 & $.839^{\prime \prime}$ & $.542^{\prime}$ & .112 & .090 & .090 \\
\hline ELUMO & $.726^{\prime \prime}$ & $.609^{\prime}$ & .227 & .004 & .416 & .518 & .317 & .140 & .403 & $.553^{\prime}$ & - 407 & 1 & $.838^{\prime \prime \prime}$ & $.547^{\prime}$ & $.861 "$ & .101 & .101 \\
\hline hardness & .423 & .414 & .330 & .233 & .388 & .306 & .130 & .038 & .155 & .366 & $.839 "$ & $.838^{\prime \prime}$ & 1 & .002 & .445 & .002 & .003 \\
\hline potential & $.682^{\prime \prime}$ & .482 & .091 & .348 & . 164 & . 481 & .381 & .199 & .500 & .451 & $.542^{\prime}$ & $.547^{\prime}$ & .002 & t & $.895^{\prime \prime}$ & .178 & -178 \\
\hline electrophilicity & $.804 "$ & $.599^{\circ}$ & .042 & .207 & .323 & $.539^{\circ}$ & .384 & .194 & .529 & $.558^{\circ}$ & .112 & $.861 "$ & .445 & $.895 "$ & T & .148 & .148 \\
\hline Hformation & .100 & .388 & .491 & $.788^{\prime \prime}$ & $.623^{\circ}$ & .371 & . 171 & 131 & .132 & $.538^{\circ}$ & .090 & .101 & .002 & . 178 & .148 & 1 & $1.000 "$ \\
\hline ETev & .100 & .388 & .491 & $.788^{\prime \prime}$ & $.623^{\circ}$ & .371 & .171 & 131 & .132 & $.538^{\prime}$ & .090 & .101 & .003 & .178 & .148 & $1.000 "$ & \\
\hline
\end{tabular}


Table 8. Results of the statistical analysis of the relationship between the values of pKa and the variables calculated by (HF) at (293 K).

\begin{tabular}{lllllll}
\hline Model & $\mathbf{R}$ & R Square & Adjusted R Square & Std. Error of the estimate & F & Sig. \\
\hline 1 & $0.998^{\mathrm{a}}$ & 0.997 & 0.979 & 0.5111011 & 57.109 & $0.017^{\mathrm{a}}$ \\
\hline
\end{tabular}

\begin{tabular}{lll}
\hline \multicolumn{1}{l}{ Coefficients } & B \\
\hline 1 & (Constant) & -50.892 \\
charge7C & -101.204 \\
chargeN9 & -43.455 \\
chargeO10 & -18.900 \\
chargeH11 & 28.943 \\
angle 2 & -.017 \\
dihedral & -.006 \\
double CN & 21.258 \\
ELUMO & 4.724 \\
potential & 5.626 \\
H formation & $-8.933 \mathrm{E}-5$ \\
charge CH3 & 35.518 \\
\hline
\end{tabular}

COMPUTE PKA $=-50.892+-101.204 *$ charge $7 \mathrm{C}+-43.455 *$ chargeN9 +-18.900*chargeO10 + $28.943 *$ chargeH1 $1+-.017 *$ angle $2+-.006 *$ dihedral $+21.258 *$ double CN +4.724*ELUMO + $5.626 *$ potential $+-8.933 \mathrm{E}-5 * \mathrm{H}$ formation $+35.518 *$ chargeCH3.

Table 9. Correlation coefficient values between variables calculated by (DFT) method at (K293).

\begin{tabular}{|c|c|c|c|c|c|c|c|c|c|c|c|c|c|c|c|c|c|}
\hline DFT 923 & charge7C & charge CH3 & chargeng & charge010 & chargeH11 & angle127 & angle 2 & dihadral & duble CN & DM & EHOMO & ELUMO & hardness & potential & electrophilicity & Hformation & ETeV \\
\hline charge7c & 1 & $.913^{111}$ & $.758^{111}$ & .030 & .395 & $.691^{\prime \prime}$ & $.600^{\circ}$ & .279 & .394 & $.660^{\circ}$ & .002 & $.860^{\prime \prime}$ & .520 & $.666^{11}$ & $.859^{11}$ & -172 & .172 \\
\hline charge CH3 & $.913^{\prime \prime}$ & 1 & $.767^{\prime \prime}$ & $\cdot 267$ & $.566^{\circ}$ & $.616^{\prime}$ & .527 & -244 & .290 & $.796^{\prime \prime}$ & -253 & $.869^{\prime \prime}$ & $.694^{\prime \prime}$ & .459 & $.856^{\prime \prime}$ & $.539^{\prime}$ & $.539^{\circ}$ \\
\hline charge 010 & .030 & .267 & .129 & 1 & $.694^{\prime \prime}$ & .041 & .015 & .023 & .137 & .480 & .366 & .178 & .349 & .168 & .164 & $.780^{\prime \prime}$ & $.780^{\prime \prime}$ \\
\hline chargeH11 & .395 & $.566^{\circ}$ & $.572^{\prime}$ & $.694^{\prime \prime}$ & 1 & .161 & .255 & .015 & -178 & $.721 "$ & -158 & $.582^{\prime}$ & -.456 & .321 & $.580^{\circ}$ & $.674^{\prime \prime}$ & $.674^{\prime \prime}$ \\
\hline angle 2 & $.600^{\circ}$ & .527 & $.689^{\prime \prime}$ & .015 & .255 & $.630^{\circ}$ & 1 & .025 & .420 & $.629^{\prime}$ & .363 & $.606^{\circ}$ & $.609^{\prime}$ & .163 & $.589^{\prime}$ & 184 & . 184 \\
\hline dihadral & .279 & $\cdot 244$ & .012 & .023 & .015 & .080 & .025 & 1 & .049 & .038 & $\cdot .267$ & -281 & .007 & .442 & .283 & .009 & .009 \\
\hline duble CN & .394 & .290 & .054 & .137 & .178 & .420 & .420 & .049 & 1 & .017 & -024 & .118 & .087 & .070 & .107 & -150 & .150 \\
\hline DM & $.660^{\circ}$ & $.796^{\prime \prime}$ & $.902^{\prime \prime}$ & .480 & $.721^{\prime \prime}$ & $.604^{\prime}$ & $.629^{\prime}$ & .038 & .017 & 1 & $.537^{\prime}$ & $.855^{\prime \prime}$ & $.874^{\prime \prime}$ & .211 & $.839^{\prime \prime}$ & $.707^{\prime \prime \prime}$ & $.707^{\prime \prime}$ \\
\hline EHOMO & .002 & .253 & .416 & .366 & .158 & .164 & .363 & .267 & .024 & $.537^{\prime}$ & 1 & .238 & $.808^{\prime \prime}$ & $.659^{\prime}$ & .189 & $.703^{\prime \prime}$ & $.703^{\prime \prime}$ \\
\hline electrophilicity & $.859^{\prime \prime}$ & $.856 "$ & $.921^{\prime \prime}$ & .164 & $.580^{\circ}$ & $.595^{\circ}$ & $.589^{\prime}$ & .283 & .107 & $.839 "$ & .189 & $.997^{\prime \prime}$ & $.730^{\prime \prime}$ & $.613^{n}$ & 1 & .414 & .414 \\
\hline Hformation & -172 & $.539^{\circ}$ & .447 & $.780^{\prime \prime}$ & $.674^{\prime \prime}$ & .109 & -184 & .009 & -150 & $.707^{\prime \prime}$ & $.703^{\prime \prime}$ & .441 & $.734^{\prime \prime}$ & .249 & .414 & 1 & $1.000^{\prime \prime}$ \\
\hline ETev & .172 & $.539^{\prime}$ & .447 & $.780^{\prime \prime}$ & $.674^{\prime \prime}$ & .109 & .184 & .009 & . 150 & $.707^{\prime \prime}$ & $.703^{\prime \prime}$ & .441 & $.734^{\prime \prime \prime}$ & .249 & .414 & $1.000^{\prime \prime}$ & 1 \\
\hline
\end{tabular}


Table 10. Results of the statistical analysis of the relationship between the values of pKa and the variables calculated by (DFT) at (293 K).

\begin{tabular}{lllllll}
\hline Model & R & R Square & Adjusted R Square & Std. Error of the Estimate & F & Sig. \\
\hline 1 & $0.988^{\mathrm{a}}$ & 0.976 & 0.691 & 1.9776005 & 3.424 & $0.401^{\mathrm{a}}$ \\
\hline
\end{tabular}

\begin{tabular}{lll}
\hline Coefficients & B \\
\hline 1 & (Constant) & 1298.882 \\
Charge7C & -62.106 \\
ChargeN9 & -21.615 \\
ChargeO10 & -44.068 \\
ChargeH11 & 18.937 \\
Angle127 & 1.547 \\
Angle 2 & -1.568 \\
Dihedral & 0.075 \\
DM & 15.362 \\
Potential & -111.888 \\
Double CN & -1076.885 \\
H formation & $5.607 \mathrm{E}-5$ \\
EHOMO & -72.439 \\
\hline
\end{tabular}

COMPUTE PKA $=1298.882+-62.106 *$ charge $7 \mathrm{C}+-21.615 *$ chargeN9 $+-44.068 *$ chargeO10 +18.937*chargeH11 +1.547*angle127 +$1.568 *$ angle $2+.075 *$ dihedral $+15.362 * \mathrm{DM}+-111.888 *$ potential $+-1076.885 *$ double $\mathrm{CN}+5.607 \mathrm{E}-5 * \mathrm{H}$ formation $+-72.439 * \mathrm{EHOMO}$.

Therefore, the values of the static constant values were the constant ionization process through the linear calculated theoretically and compared with the values of equation obtained as follows:

Table 11. The practical values, theoretical values and the amount of difference in each of the methods used.

\begin{tabular}{llllllll}
\hline $\begin{array}{l}\text { Com } \\
\text { No. }\end{array}$ & $\begin{array}{l}\text { pKa } \\
\text { practical }\end{array}$ & $\begin{array}{l}\mathbf{p K a} \\
\mathbf{A M 1}\end{array}$ & $\mathbf{\Delta \text { pKa }}$ & $\begin{array}{l}\mathbf{p K a} \\
\mathbf{D F T}\end{array}$ & $\Delta \mathbf{p K a}$ & $\begin{array}{l}\mathbf{p K a} \\
\mathbf{H F}\end{array}$ & $\Delta \mathbf{p K a}$ \\
\hline 1 & 12.666 & 12.666 & 0.000 & 12.790 & -0.124 & 12.644 & 0.023 \\
2 & 12.765 & 12.765 & 0.000 & 12.652 & 0.113 & 12.833 & -0.068 \\
3 & 6.940 & 6.940 & 0.000 & 7.690 & -0.750 & 6.756 & 0.184 \\
4 & 9.578 & 9.578 & 0.000 & 9.851 & -0.273 & 9.753 & -0.175 \\
5 & 6.398 & 6.398 & 0.000 & 6.405 & -0.007 & 6.612 & -0.214 \\
6 & 8.895 & 8.895 & 0.000 & 9.247 & -0.352 & 8.873 & 0.022 \\
7 & 11.919 & 11.919 & 0.000 & 10.879 & 1.040 & 11.925 & -0.006 \\
8 & 11.688 & 11.688 & 0.000 & 11.652 & 0.036 & 11.599 & 0.089 \\
\hline
\end{tabular}




\begin{tabular}{llllllll}
\hline $\begin{array}{l}\text { Com } \\
\text { No. }\end{array}$ & $\begin{array}{l}\mathbf{p K a} \\
\mathbf{p r a c t i c a l}\end{array}$ & $\begin{array}{l}\mathbf{p K a} \\
\mathbf{A M 1}\end{array}$ & $\mathbf{\Delta \mathbf { p K a }}$ & $\begin{array}{l}\mathbf{p K a} \\
\mathbf{D F T}\end{array}$ & $\mathbf{\Delta \mathbf { p K a }}$ & $\begin{array}{l}\mathbf{p K a} \\
\mathbf{H F}\end{array}$ & $\Delta \mathbf{p K a}$ \\
\hline 9 & 11.798 & 11.798 & 0.000 & 11.185 & 0.613 & 11.575 & 0.223 \\
10 & 12.585 & 12.585 & 0.000 & 12.424 & 0.161 & 13.101 & -0.516 \\
11 & 12.758 & 12.758 & 0.000 & 14.058 & -1.300 & 12.439 & 0.319 \\
12 & 2.990 & 2.990 & 0.000 & 3.047 & -0.057 & 2.988 & 0.002 \\
13 & 3.714 & 3.714 & 0.000 & 3.926 & -0.212 & 3.733 & -0.019 \\
14 & 5.494 & 5.494 & 0.000 & 5.559 & -0.065 & 5.565 & -0.071 \\
\hline
\end{tabular}

In considering the tables, it may be noted that

1. By studying the effect of charges in all studied amines, which represents the sensitivity of the theoretical method adopted, it was found that the AM1 and HF methods give more accurate results and correspond to the previous literature (Najim, 2010) and studies in this field.

2. The success of the study of the temporal order of all imines and the three theoretical methods used when compared with previous empirical studies (Toma AlBakzo, 2012).

3. The calculated energy variables were theoretically calculated using the three methods, and at the five thermal degrees of all imines, which represent the stability of molecules through interference and spatial changes (HOMO, LUMO, hardness, chemical electron voltage, and electrolyte guide). In the literature (Chipman, 2002; Ebead et al., 2010) related to the stability of molecules and their tendency in terms of electrophilic or nucleophile, and it was found that the values vary according to the location of the compensators and their size and spatial structure. The small values of the variable (W) The reaction of nucleoclophyl (which has the ability to give electrons), as has been demonstrated in previous studies (Al-Tayar, 2004).

4. The variables in the third paragraph were introduced in the three theoretical methods and linked with each other and with pKa values and their validity and accuracy in the statistical analysis of all the imines under study and at different temperature grades. We obtained a good match between the practical and theoretical values as well as the good correlation values close to one.

5. The values of the coefficients of the variables $\mathrm{B}$ (beta) Coefficients of the variables (C7, N9, O10, $\mathrm{H} 11, \mathrm{CH} 3$ or $\mathrm{H}$ were varied in sequence and this indicates that the variables have a clear effect in the values of $\mathrm{pKa}$ and this corresponds to what was observed in the literature (Hollingeworth et al., 2002). These variables represent the center of the interaction for the ionization constants. This indicates the validity of the selection of the variables used in this study. The standard error (SE) for the relationship between the values of the ionization constants and the variables calculated theoretically in the regression analysis and for all the compounds and the three methods was little and within acceptable ranges of experimental errors, The correlation coefficient values of the variables used For the pKa of the compounds under study in the multiple regression analysis was close to one. This indicates the importance of using these variables in calculating the value of $\mathrm{pKa}$ and comparing them with the practical values. Although the nature of these variables is different and different, most of them include the values of the interaction center as well as other values on the correlation coefficient in the regression analysis. The significant value (sig.) was significant (0.05), and the value of (F) was also high, meaning that the choice of values in the calculations was got.

\section{Conflict of interest statement}

Authors declare that they have no conflict of interest. 


\section{References}

Al-Tayar, SH. S. O., 2004. The Effect of Molecular Structure and Some Physical Properties of a Number of Conjugated Polymers on their Electrical Conductivity in the Pure and Doped States. Ph. D. Thesis, Chemistry Department, College of Science, University of Mosul.

Barlin, G. B., Perrin, D. D., 1972. Technique of Organic Chemistry. Vol.4, Part 1, Wiley Interscience, New York.

Brouwer, F., 2003. Molecular Modeling: Introduction. University of Amstrdam. [website of (Dr.F. Brouwer)].

Chipman, D.M., 2002. Computation of pKa from dielectric Quantum theory. J. Phys. Chem. A 106, 7413-7422.

da Silva, C.O., da Silva, E.C., Nascimento, M.A.C., 1999. Ab initio calculations of absolute $\mathrm{pKa}$ values in aqueous solution I. Carboxylic Acids. J. Phys. Chem. A. 103(50), 11194-11199.

Ebead, Y.H., Salman, H.M.A., Abdelah, M.A., 2010. Experimental and theoretical investigation of spectral, tautomerism and acid-base properties of
Schiff bases derived from some amino acids. Bull. Korean Chem. Soc. 31(4), 850-858.

Hollingeworth, C.A., Seybold, P.G., Hdad, C.M., 2002. Substituent effects on the electronic structure and pKa of benzoic acid. Int. J. Quantum Chem. 90, 1396-1403.

Isaacs, N. S., 1969. CNDO solutions for paraffin hydrocarbons and carbonium ions. Tetrahedron. 25, 3555-3566.

Najim, Z. A., 2010. Development of a new set of additive parameters affecting the rate of substitution of bromoalkylamine. Tikrit. J. Pure Sci. 15(1), 244-250.

Parr, R. G., Pearson, R. G., 1983. Absolute hardness: Companion parameter to absolute electronegativity. J. Amer. Chem. Soc. 105(26), 7512-7516.

Perrin, D. D., Dempsey, B., Serjeant, F. P., 1981. pKa Prediction of Organic Acids and Bases. Chapman and Hall London, Methuen, New York.

Szabo, A., Nostlund, N.S., 1989. Modern Quantum Chemistry. $1^{\text {st }}$ Edn., Dover Publication, New York.

Toma Al-Bakzo, D. B., 2012. Determination of Ionization Constants for a Number of Oximes and Schiff Bases and Studying the Factors Affecting them. M. Sc. Thesis, Mosul University.

\section{How to cite this article:}

Hussein Al-Niemi, M. M., AL-Shoraify, W. I., 2017. Statistical study of ionization for a number of oximes and Schiff bases theoretically by using molecular modeling programs. Int. J. Curr. Res. Biosci. Plant Biol. 4(8), 30-41. doi: https://doi.org/10.20546/ijcrbp.2017.408.005 